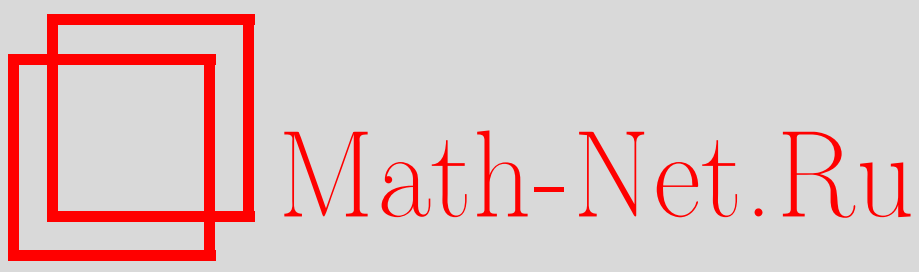

О. Е. Арсеньева, В. Ф. Кириченко, Автодуальная геометрия обобщенных эрмитовых поверхностей, Матем. сб., 1998, том 189, номер 1, 21-44

DOI: https://doi.org/10.4213/sm288

Использование Общероссийского математического портала Math-Net.Ru подразумевает, что вы прочитали и согласны с пользовательским соглашением http: //www. mathnet.ru/rus/agreement

Параметры загрузки:

IP: 54.147 .182 .235

26 апреля 2023 г., $17: 38: 32$ 
УДК 514.76

\author{
О.Е. Арсеньева, В.Ф. Кириченко
}

\title{
Автодуальная геометрия обобщенных эрмитовых поверхностей
}

В работе получен ряд результатов, касающихся геометрии конформно-полуплоских эрмитовых поверхностей как классического, так и гиперболического типа (обобщенных эрмитовых поверхностей), часть из которых обобщает и проясняет известные в этом направлении результаты Коды, Ито и других авторов. Эти результаты вскрьвают ряд красивых и неожиданных связей между такими классическими характеристиками конформно-полуплоских (обобщенных) эрмитовых поверхностей, как эйнштейновость, постоянство голоморфиной секционной кривизны, и др. Получена полная классифокикация компактных автодуальных эрмитовых $R K$-поверхностей, являющихся обобщенньми многообразиями Хопфа, даюшая полное решение проблемы Чена в этом классе эрмитовых поверхностей.

Библиографияя: 14 названий.

Эрмитовой поверхностью называется 4-мерное почти эрмитово многообразие со знакоопределенной метрикой и интегрируемой почти комплексной структурой. Если $\{M, J, g=\langle\cdot, \cdot\rangle\}$ - эрмитова поверхность, то многообразие $M$ можно рассматривать как двумерное комплексное многообразие, снабженное эрмитовой метрикой $\langle\langle X, Y\rangle\rangle=\langle X, Y\rangle+\sqrt{-1}\langle X, J Y\rangle ; X, Y \in \mathfrak{X}(M)$, (где $\mathfrak{X}(M)$ - модуль гладких векторных полей на $M)$, т.е. как комплексный аналог римановых поверхностей, чем и объясняется название. Эрмитовы поверхности изучались с различных точек зрения многими авторами (см., например, [1]-[3]). Одна из важнейших особенностей геометрии эрмитовых поверхностей, как и всяких четырехмерных псевдоримановых многообразий, определяется тем, что группа Ли $\mathrm{SO}(4, \mathbb{R})$ не является простой. Геометрическим следствием этого факта является сушествование канонического разложения модуля $\Lambda_{2}(M)$ 2-форм такого многообразия $M$ в прямую сумму подмодулей $\Lambda_{+}(M)$ автодуальных и $\Lambda_{-}(M)$ антиавтодуальных форм. Многообразие $M$ называется автодуальным (соответственно, антиавтодуальным. ), если ограничение эндоморфизма Вейля конформной кривизны на подмодуль $\Lambda_{-}(M)$ (соответственно, $\left.\Lambda_{+}(M)\right)$ равно нулю. Значение автодуальных и антиавтодуальных (иначе говоря, конформно полуплоских) многообразий определяется связью их геометрии с геометрией твисторного расслоения над такими многообразиями. Например, известная теорема Пенроуза-Атьи-Хитчина-Сингера [4] утверждает, что каноническая почти комплексная структура пространства твисторов 4-мерного ориентированного риманова многообразия $(M, g)$ интегрируема тогда и только тогда, когда $(M, g)$ конформно полуплоско. В работе [5] Чен поставил проблему классификации конформно полуплоских эрмитовых поверхностей. В направлении решения этой проблемы был получен ряд результатов [6]. Однако сушественные продвижения были получены лишш в случае келеровых поверхностей (т.е. 4-мерных келеровых многообразий). Например, Чен [5] и независимо и 
другими методами Бургињьон [7] и Дердзински [8] получили классификацию компактных автодуальных келеровых многообразий, а Ито [9] - исчерпывающее описание антиавтодуальных келеровых многообразий.

В настоящей работе получен ряд результатов, касаюшихся геометрии конформно-полуплоских эрмитовых поверхностей как классического, так и гиперболического типа (обобщенных эрмитовых поверхностей), часть из которых обобщает и проясняет известные в этом направлении результаты Коды, Ито и других авторов. Эти результаты вскрывают ряд красивых и неожиданных связей между такими классическими характеристиками конформно-полуплоских (обобщенных) эрмитовых поверхностей, как эйнштейновость, постоянство голоморфной секционной кривизны, и др. Получена полная классификация компактных автодуальных эрмитовых $R K$-поверхностей, являюшихся обобщенными многообразиями Хопфа, даюшая полное решение проблемы Чена в этом классе эрмитовых поверхностей.

Пусть $(M, g=\langle\cdot, \cdot\rangle)$ - 4-мерное ориентированное псевдориманово многообразие, $C^{\infty}(M)$ - алгебра гладких функций на $M, \mathfrak{X}(M)$ - модуль гладких векторных полей на $M, \Lambda(M)=\bigoplus_{r=0}^{\infty} \Lambda_{r}(M)$ - алгебра Грассмана дифференциальных форм на $M, *: \Lambda_{r}(M) \rightarrow \Lambda_{4-r}(M)$ - оператор Ходжа, определяемьй тождеством $\omega \wedge(* \theta)=\langle\omega, \theta\rangle \eta_{g}$, где $\omega, \theta \in \Lambda_{r}(M), \eta_{g}$ - форма объема на $M,\langle\cdot, \cdot\rangle$ - метрика на $\Lambda_{r}(M)$, индуцированная метрикой $g$. Как известно, $*^{2}=(-1)^{r(4-r)+s}$, где $s$ - отрицательный индекс инерции метрики $g$. В частности, $*: \Lambda_{2}(M) \rightarrow \Lambda_{2}(M)-$ инволюция, т.е. $*^{2}=\mathrm{id}$, тогда и только тогда, когда $s=0$ либо 2 (мы считаем, что $4-s \geqslant s)$. В этом случае $\Lambda_{2}(M)=\Lambda_{+}(M) \oplus \Lambda_{-}(M)$, где $\Lambda_{+}(M)$ и $\Lambda_{-}(M)-$ собственные подмодули эндоморфизма $*$, соответствующие собственным значениям 1 и -1 соответственно. Их элементы называются, соответственно, автодуальными и антиавтодуальными формами.

Пусть теперь $\{g, J\}$ - почти эрмитова структура классического (с положительно определенной метрикой) либо гиперболического типа, короче, обобщенная почти эрмитова, или $G A \mathscr{H}$-структура на $M, J^{2}=\alpha \mathrm{id}, \alpha=-1$ в классическом и $\alpha=1$ в гиперболическом случаях, $\langle J X, J Y\rangle=-\alpha\langle X, Y\rangle ; X, Y \in \mathfrak{X}(M)$, $\Omega(X, Y)=\left\langle J^{3} X, Y\right\rangle-$ фундаментальная форма структуры. Хорошо известно, что в гиперболическом случае $g$ - нейтральная метрика, т.е. $s=2$. Напомним [10], что 4 -мерное $G A \mathscr{H}$-многообразие, т.е. многообразие, наделенное $G A \mathscr{H}$-структурой, наделено канонической ориентацией, порожденной 4-формой $\Omega \wedge \Omega$. Как известно, задание $G A \mathscr{H}$-структуры $(g, J)$ на многообразии $M$ равносильно заданию $G$-структуры $\mathscr{G}$ со структурной группой $G=U(2, \mathfrak{K})$, где $\mathfrak{K}$ - поле $\mathbb{C}$ комплексных чисел в классическом случае либо кольцо $\mathbb{D}$ двойных чисел в гиперболическом случае. Элементы этой $G$-структуры, которую мы назовем присоединенной $G$-структурой, называются $A$-реперами [11]. Напомним построение $A$-реперов. Пусть $\left\{p, e_{1}, e_{2}, J e_{1}, J \varepsilon_{2}\right\}$ - ортонормированный репер, согласованный со структурньм эндоморфизмом $J,\left\|e_{1}\right\|^{2}=1,\left\|e_{2}\right\|^{2}=-\alpha$. Тогда соответствующий ему $A$-репер $\left\{\varepsilon_{1}, \varepsilon_{2}, \varepsilon_{\hat{1}}, \varepsilon_{\hat{2}}\right\}$ определяется как репер касательного пространства $T_{p}(M) \otimes \mathfrak{K}, p \in M$, с векторами $\varepsilon_{a}=\frac{1}{\sqrt{2}}\left(e_{a}+i J^{3} e_{a}\right), \varepsilon_{\hat{a}}=\frac{1}{\sqrt{2}}\left(e_{a}-i J^{3} e_{a}\right)$; $a, d, c, \cdots=1,2 ; \hat{a}=a+2 ; i$ - мнимая единица кольца $\mathfrak{K}$. Отметим, что $\varepsilon_{\hat{a}}=\bar{\varepsilon}_{a}$, $J \varepsilon_{a}=i \varepsilon_{a}, J \varepsilon_{\hat{a}}=-i \varepsilon_{\hat{a}}$, где $X \rightarrow \bar{X}$ - оператор естественной инволюции (сопряжения) в $\mathfrak{K}$-модуле $T_{p}(M) \otimes \mathfrak{K}$. Легко проверить также [11], что на пространстве 
$G$-структуры

$$
\left(g_{j k}\right)=\left(g^{j k}\right)=\left(\begin{array}{cccc}
0 & 0 & 1 & 0 \\
0 & 0 & 0 & -\alpha \\
1 & 0 & 0 & 0 \\
0 & -\alpha & 0 & 0
\end{array}\right) ; \quad\left(J_{k}^{j}\right)=\left(\begin{array}{cccc}
i & 0 & 0 & 0 \\
0 & i & 0 & 0 \\
0 & 0 & -i & 0 \\
0 & 0 & 0 & -i
\end{array}\right)
$$

$(j, k=1,2, \hat{1}, \hat{2})$.

Лемма 1 (см. [10]). Пусть $(M, g, J)$-4-мерное обобщенное почти эрмитово многообразие, $\theta \in \Lambda_{2}(M)$. В этом случае

1) $\theta \in \Lambda_{+}(M)$ тогда и только тогда, когда на пространстве $\mathscr{G}$

$$
\left(\theta_{j k}\right)=\left(\begin{array}{cccc}
0 & x+i z & i y & 0 \\
-x-i z & 0 & 0 & -i \alpha y \\
-i y & 0 & 0 & x-i z \\
0 & i \alpha y & -x+i z & 0
\end{array}\right)
$$

2) $\theta \in \Lambda_{-}(M)$ тогда и только тогда, когда на пространстве $\mathscr{G}$

$$
\left(\theta_{j k}\right)=\left(\begin{array}{cccc}
0 & 0 & i y & x+i z \\
0 & 0 & -x+i z & i \alpha y \\
-i y & x-i z & 0 & 0 \\
-x-i z & -i \alpha y & 0 & 0
\end{array}\right) .
$$

ОПРЕДЕЛЕНИЕ 1.4 -мерное $G A \mathscr{H}$-многообразие с интегрируемой структурой называется обобщенной эрмитовой поверхностью. Здесь интегрируемость структуры понимается как обращение в нуль тензора Нейенхейса

$$
N(X, Y)=J^{2}[X, Y]+[J X, J Y]-J[J X, Y]-J[X, J Y] ; \quad X, Y \in \mathfrak{X}(M) .
$$

Обобшенные эрмитовы многообразия. Пусть $(M, g, J)$ - почти эрмитово многообразие, $\nabla$ - риманова связность метрики $g$. Ее структурные уравнения на пространстве расслоения реперов над $M$ имеют вид:

$$
\begin{aligned}
& \text { 1) } d \omega^{i}=\omega_{j}^{i} \wedge \omega^{j} \\
& \text { 2) } d \omega_{j}^{i}=\omega_{k}^{i} \wedge \omega_{j}^{k}+\frac{1}{2} R_{j k l}^{i} \omega^{k} \wedge \omega^{l},
\end{aligned}
$$

где $\left\{\omega^{i}\right\}$ и $\left\{\omega_{j}^{i}\right\}$ - компоненты форм смещения и римановой связности соответственно, $\left\{R_{j k l}^{i}\right\}$-компоненты тензора $R$ Римана-Кристоффеля; $i, j, k, l=1, \ldots, \operatorname{dim} M$. Заметим, что в силу вешественности форм смешения и связности, а также тензора $R$, верны соотношения:

$$
\bar{\omega}^{i}=\omega^{\hat{i}} ; \quad \bar{\omega}_{j}^{i}=\omega_{\hat{j}}^{\hat{i}} ; \quad \bar{R}_{j k l}^{i}=R_{\hat{j} \hat{k} \hat{l}}^{\hat{i}} ; \quad \hat{i}=i \pm \frac{1}{2} \operatorname{dim} M
$$


Далее, поскольку $J$ и $g$ - тензоры типов $(1,1)$ и $(2,0)$ соответственно, причем $\nabla g=0$ по определению римановой связности, компоненты этих тензоров как функции на пространстве расслоения реперов над $M$ удовлетворяют системе дифференциальных уравнений

$$
\begin{aligned}
& \text { 1) } d J_{j}^{i}+J_{k}^{i} \omega_{j}^{k}-J_{j}^{k} \omega_{k}^{i}=J_{j, k}^{i} \omega^{k} \\
& \text { 2) } d g_{i j}+g_{k j} \omega_{i}^{k}+g_{i k} \omega_{j}^{k}=0
\end{aligned}
$$

где $\left\{J_{j, k}^{i}\right\}$ - компоненты тензора $\nabla J$. С учетом соотношений (1) уравнения $\left(4_{1}\right)$ и $\left(4_{2}\right)$ на пространстве присоединенной $G$-структуры примут вид, соответственно:

$$
\begin{aligned}
& \text { 1) } \left.\left.\omega_{\hat{b}}^{a}=\frac{i^{3}}{2} J_{\hat{b}, k}^{a} \omega^{k}, \quad 2\right) \omega_{b}^{\hat{a}}=-\frac{i^{3}}{2} J_{b, k}^{\hat{a}} \omega^{k}, \quad 3\right) J_{b, k}^{a}=J_{\hat{b}, k}^{\hat{a}}=0 \\
& \text { 1) } \left.\left.\varepsilon(a) \omega_{\hat{b}}^{a}+\varepsilon(b) \omega_{\hat{a}}^{b}=0, \quad 2\right) \varepsilon(a) \omega_{b}^{\hat{a}}+\varepsilon(b) \omega_{a}^{\hat{b}}=0, \quad 3\right) \varepsilon(a) \omega_{b}^{a}+\varepsilon(b) \omega_{\hat{a}}^{\hat{b}}=0
\end{aligned}
$$

$\left(a, b, c, d, \cdots=1, \ldots, \frac{1}{2} \operatorname{dim} M ; \hat{a}=a+\frac{1}{2} \operatorname{dim} M\right)$. Подставим (5) в $\left(3_{1}\right):$

$$
\begin{aligned}
& d \omega^{a}=\omega_{b}^{a} \wedge \omega^{b}+\frac{i^{3}}{2} J_{\hat{b}, c}^{a} \omega^{c} \wedge \omega^{\hat{b}}+\frac{i^{3}}{2} J_{[\hat{b}, \hat{c}]}^{a} \omega^{\hat{c}} \wedge \omega^{\hat{b}} \\
& d \omega^{\hat{a}}=\omega_{\hat{b}}^{\hat{a}} \wedge \omega^{\hat{b}}-\frac{i^{3}}{2} J_{b, \hat{c}}^{\hat{a}} \omega^{\hat{c}} \wedge \omega^{b}-\frac{i^{3}}{2} J_{[b, c]}^{\hat{a}} \omega^{c} \wedge \omega^{b}
\end{aligned}
$$

где по индексам, заключенным в квадратные скобки, подразумевается альтернирование. Введем обозначение $\omega_{i}=g_{i j} \omega^{j}$. В частности, на пространстве присоединенной $G$-структуры $\omega_{a}=\varepsilon(a) \omega^{\hat{a}}=\varepsilon(a) \bar{\omega}^{a}$. С учетом этого и соотношений $(6)$ уравнения (7) можно переписать в форме

$$
\begin{aligned}
& d \omega^{a}=\omega_{b}^{a} \wedge \omega^{b}+B^{a b}{ }_{c} \omega^{c} \wedge \omega_{b}+B^{a b c} \omega_{b} \wedge \omega_{c}, \\
& d \omega_{a}=-\omega_{a}^{b} \wedge \omega_{b}+B_{a b}{ }^{c} \omega_{c} \wedge \omega^{b}+B_{a b c} \omega^{b} \wedge \omega^{c}
\end{aligned}
$$

где

$$
\begin{aligned}
B_{c}^{a b} & =\frac{i^{3}}{2} \varepsilon(b) J_{\hat{b}, c}^{a}, \quad B^{a b c}=-\frac{i^{3}}{2} \varepsilon(b, c) J_{[\hat{b}, \hat{c}]}^{a}, \\
B_{a b}{ }^{c} & =-\frac{i^{3}}{2} \varepsilon(a, c) J_{b, \hat{c}}^{\hat{a}}, B_{a b c}=\frac{i^{3}}{2} \varepsilon(a) J_{[b, c]}^{\hat{a}} .
\end{aligned}
$$

Системы функций $\left\{B^{a b}{ }_{c}\right\},\left\{B_{a b}{ }^{c}\right\},\left\{B^{a b c}\right\},\left\{B_{a b c}\right\}$ являются компонентами комплексных тензоров на многообразии $M$, называемых, соответственно, виртуальнылм тензором первого рода, виртуальным тензором второго рода, структурным тензором первого рода и структурным тензором второго рода $G A \mathscr{H}$-многообразия $M$. Заметим, что согласно (6) $\varepsilon(a) J_{\hat{b}, k}^{a}+\varepsilon(b) J_{\hat{a}, k}^{b}=0$. Умножая обе части этого равенства на $\varepsilon(a, b)$, получим: $\varepsilon(b) J_{\hat{b}, k}^{a}+\varepsilon(a) J_{\hat{a}, k}^{b}=0$, и следовательно, $B^{a b}{ }_{c}+B^{b a}{ }_{c}=0$. Аналогично, $B_{a b}{ }^{c}+B_{b a}{ }^{c}=0$. Тем самым доказано

ПРЕДЛОЖЕНИЕ 1. Виртуальнье тензорь GАН̈-структурь кососимметричны по первой паре аргументов. 
Замечание 1 . Очевидно, что структурные тензоры $G A \mathscr{H}$-структуры кососимметричны по второй паре аргументов.

ЗАмЕчАниЕ 2 . В силу вешественности тензора $\nabla J$ из приведенных определений следует, что $\bar{B}_{c}^{a b}=\varepsilon(a, b, c) B_{a b}^{c}, \bar{B}^{a b c}=\varepsilon(a, b, c) B_{a b c}$.

ЗАмечание 3 . С учетом $\left(5_{1}\right),\left(5_{2}\right)$ и $(9)$ имеем:

$$
\omega_{\hat{b}}^{a}=\varepsilon(b) B^{a b}{ }_{c} \omega^{c} ; \quad \omega_{b}^{\hat{a}}=\varepsilon(a) B_{a b}{ }^{c} \omega_{c} .
$$

Далее, в силу отсутствия кручения римановой связности

$$
[X, Y]=\nabla_{X} Y-\nabla_{Y} X, \quad X, Y \in \mathfrak{X}(M) .
$$

Следовательно, соотношение (2) можно переписать в форме

$$
N(X, Y)=\nabla_{J X}(J) Y-\nabla_{J Y}(J) X-J \nabla_{X}(J) Y+J \nabla_{Y}(J) X .
$$

Более того, ковариантно дифференцируя соотношение $J^{2}=\alpha$ id, получим, что $J \nabla_{X}(J) Y+\nabla_{X}(J)(J Y)=0, X, Y \in \mathfrak{X}(M)$, и следовательно,

$$
N(X, Y)=\nabla_{J X}(J) Y+\nabla_{X}(J)(J Y)-\nabla_{J Y}(J) X-\nabla_{Y}(J)(J X) .
$$

Из этой формулы следует, в частности, что

$$
\begin{aligned}
N_{b c}^{\hat{a}} & =\left(\nabla_{J \varepsilon_{b}}(J) \varepsilon_{c}\right)^{\hat{a}}+\left(\nabla_{\varepsilon_{b}}(J)\left(J \varepsilon_{c}\right)\right)^{\hat{a}}-\left(\nabla_{J \varepsilon_{c}}(J) \varepsilon_{b}\right)^{\hat{a}}-\left(\nabla_{\varepsilon_{c}}(J)\left(J \varepsilon_{b}\right)^{\hat{a}}\right. \\
& =2 i\left(J_{c, b}^{\hat{a}}-J_{b, c}^{\hat{a}}\right)=-4 i J_{[b, c]}^{\hat{a}}=-8 \alpha \varepsilon(a) B_{a b c} .
\end{aligned}
$$

Аналогично, $N_{\hat{b} \hat{c}}^{a}=-8 \alpha \varepsilon(b, c) B^{a b c}$. Остальные компоненты тензора Нейенхейса на пространстве присоединенной $G$-структуры равны нулю. Тем самьм доказано

ПРЕДЛОЖЕНИЕ 2. Обобщенная почти әрмитова структура интегрируема тогда и только тогда, когда ее структурные тензоры - нулевые.

ОПРЕДЕЛЕНИЕ 2. Интегрируемая обобщенная почти эрмитова структура называется обобщенной әрмитовой (короче, $G \mathscr{H}$-) структурой.

C учетом предложения 2 и (8) первая группа структурных уравнений обобщенной эрмитовой структуры на пространстве присоединенной $G$-структуры имеет вид:

$$
\begin{aligned}
& \text { 1) } d \omega^{a}=\omega_{b}^{a} \wedge \omega^{b}+B^{a b}{ }_{c} \omega^{c} \wedge \omega_{b}, \\
& \text { 2) } d \omega_{a}=-\omega_{a}^{b} \wedge \omega_{b}+B_{a b}{ }^{c} \omega_{c} \wedge \omega^{b} .
\end{aligned}
$$

Для нахождения второй группы структурных уравнений $G \mathscr{H}$-структуры продифференцируем внешним образом соотношения (10).

Дифференцируя внешшим образом $\left(10_{1}\right)$, получим:

$$
\begin{aligned}
\Delta \omega_{b}^{a} \wedge \omega^{b} & +\Delta B^{a b}{ }_{c} \wedge \omega^{c} \wedge \omega_{b} \\
& -B^{a[b}{ }_{h} B^{|h| d]}{ }_{c} \omega^{c} \wedge \omega_{b} \wedge \omega_{d}+B^{a h}{ }_{[c} B^{d}{ }_{|h| b]} \omega^{c} \wedge \omega^{b} \wedge \omega_{d}=0,
\end{aligned}
$$


где $\Delta \omega_{b}^{a}=d \omega_{b}^{a}-\omega_{c}^{a} \wedge \omega_{b}^{c}, \Delta B^{a b}{ }_{c}=d B^{a b}{ }_{c}-B^{h b}{ }_{c} \omega_{h}^{a}-B^{a h}{ }_{c} \omega_{h}^{b}+B^{a b}{ }_{h} \omega_{c}^{h}$. Разложим формы $\Delta \omega_{b}^{a}$ и $\Delta B^{a b}{ }_{c}$ по естественным базисам:

$$
\begin{aligned}
& \text { 1) } \Delta \omega_{b}^{a}=A_{b c h}^{a d r} \omega_{d}^{c} \wedge \omega_{r}^{h}+A_{b c r}^{a d} \omega_{d}^{c} \wedge \omega^{r}+A_{b c}^{a d r} \omega_{d}^{c} \wedge \omega_{r} \\
& +A_{b c d}^{a} \omega^{c} \wedge \omega^{d}+A_{d c}^{a d} \omega^{c} \wedge \omega_{d}+A_{b}^{a c d} \omega_{c} \wedge \omega_{d}, \\
& \text { 2) } \Delta B^{a b}{ }_{c}=B^{a b}{ }_{c d}^{r} \omega_{r}^{d}+B^{a b}{ }_{c d} \omega^{d}+B^{a b}{ }_{c}{ }^{d} \omega_{d} \text {. }
\end{aligned}
$$

Подставляя эти соотношения в (11), с учетом линейной независимости базисных форм получим:

$$
\begin{gathered}
\text { 1) } A_{b c h}^{a d r}=0, \text { 2) } A_{[b|d| r]}^{a c}=0, \text { 3) } A_{b d}^{a c r}-B^{a r c}{ }_{b d}^{c}=0, \text { 4) } A_{[b c d]}^{a}=0, \\
\text { 5) } A_{[b c]}^{a d d}-B^{a d}{ }_{[b c]}+B^{a h}{ }_{[b} B_{|h| c]}{ }^{d}=0 \\
\text { 6) } A_{b}^{a c d}+B^{a\left[c_{b} d\right]}-B^{a\left[{ }_{h} B^{|h| d]}{ }_{b}\right.}=0 .
\end{gathered}
$$

Дифференцируя внешним образом $\left(10_{2}\right)$, получим:

$$
\begin{aligned}
-\Delta \omega_{a}^{b} \wedge \omega_{b}+\Delta B_{a b}^{c} \wedge \omega_{c} \wedge \omega^{b} \\
\quad-B_{a[b}{ }^{h} B_{|h| d]}{ }^{c} \omega_{c} \wedge \omega^{b} \wedge \omega^{d}+B_{a h}{ }^{[c} B^{|h| b]}{ }_{d} \omega_{c} \wedge \omega_{b} \wedge \omega^{d}=0,
\end{aligned}
$$

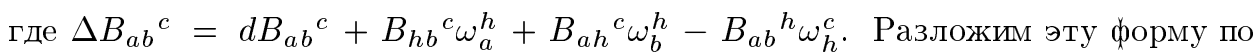
естественному базису:

$$
\Delta B_{a b}{ }^{c}=B_{a b}{ }_{r}^{c d} \omega_{d}^{r}+B_{a b}^{c}{ }_{d} \omega^{d}+B_{a b}{ }^{c d} \omega_{d} .
$$

Подставляя это разложение и $\left(12_{1}\right)$ с учетом (13) в (14), в силу линейной независимости базисных форм получим:

$$
\begin{aligned}
& A_{a d r}^{b c}+B_{a r}^{b c} d=0, \quad A_{a}^{[b c d]}=0, \\
& -A_{a c d}^{b}+B_{a\left[c^{b} d\right]}^{b}-B_{a\left[c^{h} B_{|h| d]}^{b}\right.}^{b}=0, \quad-A_{a c}^{[b d]}+B_{a c}^{[b d]}-B_{a h^{[b}} B^{|h| d]}{ }_{c}=0 .
\end{aligned}
$$

С учетом первого из этих соотношений, соотношения $\left(13_{2}\right)$ и предложения 1 получаем, что $B_{a r}{ }_{d}^{b c}=0, A_{a d r}^{b c}=0$. С учетом замечания 2 и соотношения $\left(13_{3}\right)$ получаем также, что $B^{a r} b_{c}^{d}=0, A_{b c}^{a d r}=0$. Таким образом, вторая группа структурных уравнений $G \mathscr{H}$-структуры на пространстве присоединенной $G$-структуры имеет вид:

$$
d \omega_{b}^{a}=\omega_{c}^{a} \wedge \omega_{b}^{c}+A_{b c}^{a d} \omega_{c} \wedge \omega_{d}+A_{b c d}^{a} \omega^{c} \wedge \omega^{d}+A_{b}^{a c d} \omega_{c} \wedge \omega_{d}
$$

Кроме того, применяя к обеим частям этого равенства оператор комплексного сопряжения и используя вешественность форм смешения и связности, с учетом $\left(6_{3}\right)$ и линейной независимости базисных форм получим, что

$$
\bar{A}_{b c}^{a d}=\varepsilon(a, b, c, d) A_{a d}^{b c}, \quad \bar{A}_{b}^{a c d}=-\varepsilon(a, b, c, d) A_{a c d}^{b} .
$$

Собирая полученные данные, мы приходим к следующему результату. 
ТЕОРемА 1. Полная группа структурных уравнений обобщенной әрмитовой структурь на пространстве присоединенной G-структуры имеет вид:

1) $d \omega^{a}=\omega_{b}^{a} \wedge \omega^{b}+B^{a b}{ }_{c} \omega^{c} \wedge \omega_{b}$,

2) $d \omega_{a}=-\omega_{a}^{b} \wedge \omega_{b}+B_{a b}{ }^{c} \omega_{c} \wedge \omega^{b}$,

3) $d \omega_{b}^{a}=\omega_{c}^{a} \wedge \omega_{b}^{c}+A_{b c}^{a d} \omega^{c} \wedge \omega_{d}+A_{b c d}^{a} \omega^{c} \wedge \omega^{d}+A_{b}^{a c d} \omega_{c} \wedge \omega_{d}$.

При этом

1) $d B^{a b}{ }_{c}-B^{h b}{ }_{c} \omega_{h}^{a}-B^{a h}{ }_{c} \omega_{h}^{b}+B^{a b}{ }_{h} \omega_{c}^{h}=B^{a b}{ }_{c h} \omega^{h}+B^{a b}{ }_{c}{ }^{h} \omega_{h}$,

2) $d B_{a b}{ }^{c}+B_{h b}{ }^{c} \omega_{a}^{h}+B_{a h}{ }^{c} \omega_{b}^{h}-B_{a b}{ }^{h} \omega_{h}^{c}=B_{a b}{ }^{c h} \omega_{h}+B_{a b}{ }^{c} \omega^{h}$;

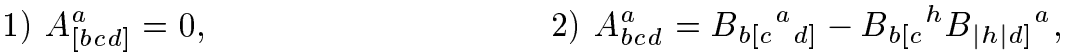

3) $A_{a}^{[b c d]}=0, \quad$ 4) $A_{a}^{b c d}=-B^{b\left[c_{a} d\right]}+B^{b\left[c_{h} B^{|h| d]}\right.}{ }_{a}$,

5) $A_{[b c]}^{a d}=B_{[b c]}^{a d}-B_{[b}^{a h} B_{|h| c]}{ }^{d}$,

6) $A_{a d}^{[b c]}=B_{a d}[b c]-B_{a h}^{[b} B^{|h| c]} d$.

Вычислим компоненты тензора $R$ на пространстве присоединенной $G$-структуры. С одной стороны, положив в $\left(3_{2}\right) i=a, j=b$, с учетом замечания 3 получим:

$$
\begin{aligned}
d \omega_{b}^{a} & =\omega_{c}^{a} \wedge \omega_{b}^{c}+\omega_{\hat{c}}^{a} \wedge \omega_{b}^{\hat{c}}+\frac{1}{2} R_{b i j}^{a} \omega^{i} \wedge \omega^{j} \\
& =\omega_{c}^{a} \wedge \omega_{b}^{c}+B^{a h}{ }_{c} B_{h b}{ }^{d} \omega^{c} \wedge \omega_{d}+\frac{1}{2} R^{a}{ }_{b i j} \omega^{i} \wedge \omega^{j} .
\end{aligned}
$$

Сравнивая с $\left(15_{3}\right)$, с учетом линейной независимости базисных форм получим: $R_{b c d}^{a}=2 A_{b c d}^{a}, R_{b c}^{a}+B^{a h}{ }_{c} B_{h b}{ }^{d}=A_{b c}^{a d}$, и значит,

$$
\text { 1) } R_{\hat{a} b c d}=2 \varepsilon(a) A_{b c d}^{a}, \quad \text { 2) } R_{\hat{a} b c \hat{d}}=\varepsilon(a, d)\left(A_{b c}^{a d}-B^{a h}{ }_{c} B_{h b}{ }^{d}\right) \text {. }
$$

Далее, дифференцируя внешним образом соотношение $\omega_{b}^{\hat{a}}=\varepsilon(a) B_{a b}{ }^{c} \omega_{c}$, с учетом $\left(3_{2}\right),\left(16_{2}\right)$ и $\left(15_{2}\right)$ получим:

$$
\begin{aligned}
& \omega_{c}^{\hat{a}} \wedge \omega_{b}^{c}+\omega_{\hat{c}}^{\hat{a}} \wedge \omega_{b}^{\hat{c}}+\frac{1}{2} R_{b i j}^{\hat{a}} \omega^{i} \wedge \omega^{j} \\
& =\varepsilon(a)\left\{-B_{h b}{ }^{c} \omega_{a}^{h}-B_{a h}{ }^{c} \omega_{b}^{h}+B_{a b}{ }^{h} \omega_{h}^{c}+B_{a b}{ }^{c}{ }_{h} \omega^{h}+B_{a b}{ }^{[c h]} \omega_{h}\right\} \wedge \omega_{c} \\
& +\varepsilon(a) B_{a b}^{c}\left(-\omega_{c}^{d} \wedge \omega_{d}+B_{c d}^{r} \omega_{r} \wedge \omega^{d}\right) \text {. }
\end{aligned}
$$

Сделаем необходимые сокращения с учетом замечания 3 и формулы $\left(6_{3}\right)$ :

$$
\frac{1}{2} R^{\hat{a}}{ }_{b i j} \omega^{i} \wedge \omega^{j}=\varepsilon(a)\left\{\left(-B_{a b}{ }^{h} B_{h c}{ }^{d}+B_{a b}{ }^{d}{ }_{c}\right) \omega^{c} \wedge \omega_{d}-B_{a b}[c d] \omega_{c} \wedge \omega_{d}\right\} .
$$

Ввиду линейной независимости базисных форм находим отсюда, что $R^{\hat{a}} b c d=0$, $R_{b}^{\hat{a}_{b} c d}=-2 \varepsilon(a) B_{a b}{ }^{[c d]}$. Заметим, что операция сопряжения позволяет последнюю формулу переписать в виде $R_{\hat{a} \hat{b} c d}=-2 \varepsilon(a, b) B^{a b}{ }_{[c d]}$. Таким образом,

$$
\text { 1) } R_{a b c d}=0, \text { 2) } R_{\hat{a} \hat{b} c d}=-2 \varepsilon(a, b) B^{a b}{ }_{[c d]} \text {. }
$$


Остальные компоненты тензора $R$ получаются с учетом вешественности этого тензора и его классических свойств симметрии.

Заметим, что из (19) следует также, что $R_{a b c \hat{d}}=-B_{a b}{ }^{h} B_{h c}{ }^{d}+B_{a b}{ }^{d}$. С учетом классических свойств симметрии тензора $R$ это соотношение можно переписать в форме $R_{\hat{a} b c d}=B_{c d}{ }^{h} B_{h b}{ }^{a}+B_{d c}{ }^{a}{ }_{b}$. С учетом (18 1$)$ имеем отсюда:

$$
A_{b c d}^{a}=\frac{1}{2}\left(B_{c d}{ }^{h} B_{h b}{ }^{a}-B_{c d}{ }^{a} b\right) .
$$

Выгислим теперь компоненты тензора Риччи $r G \mathscr{H}$-многообразия на пространстве присоединенной $G$-структуры. Имеем по определению: $r_{i j}=R^{k}{ }_{i j k}$, и значит, в частности:

$$
\text { 1) } \begin{aligned}
r_{a b} & =R_{a b c}^{c}+R_{a b \hat{c}}^{\hat{d}}=\sum_{c=1}^{2} \varepsilon(c)\left(R_{\hat{c} a b c}+R_{\hat{c} b a c}\right)=2\left(A_{a b c}^{c}+A_{b a c}^{c}\right) \\
& =B_{b c}{ }^{h} B_{h a}{ }^{c}+B_{a c}{ }^{h} B_{h b}{ }^{c}-B_{b c^{c}{ }^{c} a}-B_{a c}{ }^{c} b=2\left(B_{c\left(a^{h}\right.}{ }^{h} B_{b) h}{ }^{c}+B_{c(a b)}{ }^{c} b\right),
\end{aligned}
$$

где по индексам, заключенным в скобки, подразумевается симметризация,

$$
\text { 2) } \begin{aligned}
r_{a \hat{b}} & =R_{a \hat{b} c}^{c}+R_{a \hat{b} \hat{b}}^{\hat{c}} \\
& =\sum_{c=1}^{2} \varepsilon(c)\left(R_{\hat{c} a \hat{b} b}+R_{c a \hat{b} \hat{c}}\right)=\sum_{c=1}^{2} \varepsilon(c)\left(R_{\hat{c} \hat{b} a c}-R_{\hat{c} a c \hat{b}}\right) \\
& =-\varepsilon(b)\left(2 B^{c b}{ }_{[a c]}+A_{a c}^{c b}-B^{c h}{ }_{c} B_{h a}{ }^{b}\right) \\
& =\varepsilon(b)\left\{2 B^{c b}{ }_{[c a]}-\left(A_{c a}^{c b}+2 B^{c b}{ }_{[a c]}-2 B^{c h}{ }_{[a} B_{|h| c]}{ }^{b}\right)+B^{c h}{ }_{c} B_{h a}{ }^{b}\right\} \\
& =\varepsilon(b)\left(-A_{c a}^{c b}+4 B^{c b}{ }_{[c a]}-B^{c h}{ }_{a} B_{c h}{ }^{b}\right) .
\end{aligned}
$$

Таким образом, с учетом вешественности тензора Риччи имеем

$$
\begin{aligned}
& \left.r_{a b}=2\left(B_{c(a}{ }^{h} B_{b) h}{ }^{c}+B_{c(a}{ }^{c} b\right)\right), \\
& \left.r_{\hat{a} \hat{b}}=2 \varepsilon(a, b)\left(B^{c(a}{ }_{h} B^{b) h}{ }_{c}+B^{c(a}{ }_{c} b\right)\right), \\
& r_{a \hat{b}}=r_{\hat{b} a}=\varepsilon(b)\left(-A_{c a}^{c b}+4 B_{[c a]}^{c b}-B_{a}^{c h} B_{c h}{ }^{b}\right) .
\end{aligned}
$$

Вычислим, наконец, компоненты тензора $W$ Вейля конформной кривизны $G \mathscr{H}$-многообразия на пространстве присоединенной $G$-структуры. Напомним [10], что компоненты этого тензора вычисляются по формуле

$W_{i j k m}=R_{i j k m}+\frac{1}{2}\left(r_{i k} g_{j m}+r_{j m} g_{i k}-r_{i m} g_{j k}-r_{j k} g_{i m}\right)+\frac{\varkappa}{6}\left(g_{i m} g_{j k}-g_{i k} g_{j m}\right)$, где $\varkappa=g^{i j} r_{i j}$ - скалярная кривизна многообразия. С учетом этого, а также соотношений (18) и (20) получаем:

1) $W_{a b c d}=0$,

2) $W_{\hat{a} b c d}=2 \varepsilon(a) A_{b c d}^{a}+\frac{1}{2}\left(r_{b d} g_{\hat{a} c}-r_{b c} g_{\hat{a} d}\right)$,

3) $W_{\hat{a} \hat{b} c d}=-2 \varepsilon(a, b) B^{a b}{ }_{[c d]}+\frac{1}{2}\left(r_{\hat{a} c} g_{\hat{b} d}+r_{\hat{b} d} g_{\hat{a} c}-r_{\hat{a} d} g_{\hat{b} c}-r_{\hat{b} c} g_{\hat{a} d}\right)$

$$
+\frac{\varkappa}{6}\left(g_{\hat{a} d} g_{\hat{b} c}-g_{\hat{a} c} g_{\hat{b} d}\right),
$$

4) $W_{\hat{a} b c \hat{d}}=\varepsilon(a, d)\left\{A_{b c}^{a d}-B^{a h}{ }_{c} B_{h b}{ }^{d}\right\}+\frac{1}{2}\left(r_{\hat{a} c} g_{b \hat{d}}+r_{b \hat{d}} g_{\hat{a} c}\right)-\frac{\varkappa}{6} g_{\hat{a} c} g_{b \hat{d}}$. 
Остальные компоненты тензора $W$ получаются с учетом вещественности этого тензора и его свойств симметрии, аналогичных свойствам симметрии тензора $R$.

Обобщенные эрмитовы поверхности. Пусть $(M, g, J)$ - обобщенная эрмитова поверхность. Тогда индексы $a, b, c, d, \ldots$ пробегают значения 1 и 2 . Введем в рассмотрение тензор $C$ с компонентами $C^{a b}{ }_{c}=B^{a b}{ }_{c}+2 B^{h[a}{ }_{h} \delta_{c}^{b]}$. Непосредственно проверяется, что $C^{12}{ }_{1}=C^{12}{ }_{2}=0$, и значит, $C^{a b}{ }_{c}=0$, т.е. $B^{a b}{ }_{c}=-2 B^{h[a}{ }_{h} \delta_{c}^{b]}$. Введем в рассмотрение функции $\alpha^{a}=-2 B^{h a}{ }_{h}$. Тогда $B^{a b}{ }_{c}=\alpha^{[a} \delta_{c}^{b]}$. Аналогично, $B_{a b}{ }^{c}=\alpha_{[a} \delta_{b]}^{c}$, где $\alpha_{a}=-2 B_{h a}{ }^{h}$. Итак,

$$
B^{a b}{ }_{c}=\alpha^{[a} \delta_{c}^{b]}, \quad B_{a b}^{c}=\alpha_{[a} \delta_{b]}^{c} .
$$

Свернем это соотношение по индексам $b$ и $c$ :

$$
\alpha^{a}=2 B_{c}^{a c}, \quad \alpha_{a}=2 B_{a c}^{c} .
$$

С учетом $(9)$ и $\left(5_{3}\right)$ получим:

$$
\alpha^{a}=i^{3} \sum_{c=1}^{2} \varepsilon(c) J_{\hat{c}, c}^{a}=i^{3} J_{\hat{b}, c}^{a} g^{\hat{b} c}=i^{3} J_{j, k}^{a} g^{j k} .
$$

$\mathrm{C}$ другой стороны, по определению имеем: $\Omega(X, Y)=\left\langle J^{3} X, Y\right\rangle=\alpha\langle J X, Y\rangle$, и значит, $\nabla_{Z}(\Omega)(X, Y)=\alpha\left\langle\nabla_{Z}(J) X, Y\right\rangle$, откуда $\Omega_{i j, k}=\alpha J_{i, k}^{r} g_{r j}$ или

$$
\Omega_{i j, k}=-\alpha g_{i r} J_{j, k}^{r} .
$$

Следовательно, $(\delta \Omega)_{i}=\Omega_{i j, k} g^{j k}=-\alpha g_{i r} g^{j k} J_{j, k}^{r}$, где $\delta$ - оператор кодифференцирования. В частности, на пространстве присоединенной $G$-структуры $(\delta \Omega)_{\hat{a}}=$ $-\alpha g_{\hat{a} b} g^{j k} J_{j, k}^{b}=-i^{2} \varepsilon(a) J_{j, k}^{a} g^{j k}$, и следовательно, с учетом (1)

$$
(\delta \Omega \circ J)_{\hat{a}}=-i(\delta \Omega)_{\hat{a}}=\varepsilon(a) i^{3} J_{j, k}^{a} g^{j k}=\varepsilon(a) \alpha^{a}=\alpha_{\hat{a}} .
$$

Аналогично, $(\delta \Omega \circ J)_{a}=\alpha_{a}$ и, таким образом, функции $\left\{\alpha_{a}, \alpha_{\hat{a}}\right\}$ являются компонентами 1-формы $\omega=\delta \Omega \circ J$, называемой формой Ли многообразия. Тем самым доказано

ПРЕДЛОЖЕНИЕ 3. Первая группа структурных уравнений обобщенной әрмитовой поверхности на пространстве присоединенной $G$-структуры имеет вид

$$
\begin{aligned}
d \omega^{a} & =\omega_{b}^{a} \wedge \omega^{b}+B^{a b}{ }_{c} \omega^{c} \wedge \omega_{b}, \\
d \omega_{a} & =-\omega_{a}^{b} \wedge \omega_{b}+B_{a b}{ }^{c} \omega_{c} \wedge \omega^{b},
\end{aligned}
$$

причем виртуальные тензори имеют строение $B^{a b}{ }_{c}=\alpha^{[a} \delta_{c}^{b]}, B_{a b}{ }^{c}=\alpha_{[a} \delta_{b]}^{c}$, где функции $\left\{\alpha_{a}, \alpha_{\hat{a}}\right\}$ на пространстве присоединенной $G$-структуры являются компонентами формы Ли әрмитовой поверхности.

Свернем (16) по индексам $b$ и $c$. С учетом (25) получим:

$$
\begin{aligned}
d \alpha^{a}-\alpha^{b} \omega_{b}^{a} & =\alpha^{a}{ }_{h} \omega^{h}+\alpha^{a h} \omega_{h}, \\
d \alpha_{a}-\alpha_{b} \omega_{a}^{b} & =\alpha_{a}{ }^{h} \omega_{h}+\alpha_{a h} \omega^{h} .
\end{aligned}
$$


Дифференцируя с учетом (16) и (26) соотношения (24), ввиду линейной независимости базисных форм получим:
1) $B^{a b}{ }_{c d}=\alpha^{[a}{ }_{d} \delta_{c}^{b]}$,
2) $B^{a b}{ }_{c}{ }^{d}=\alpha^{[a|d|} \delta_{c}^{b]}$,
3) $B_{a b}{ }^{c d}=\alpha_{[a}{ }^{d} \delta_{b]}^{c}$,
4) $B_{a b}{ }^{c}{ }_{d}=\alpha_{[a|d|} \delta_{b]}^{c}$.

ЗАмЕчАнИЕ 4. Непосредственно проверяется, что поскольку комплексная размерность обобщенной эрмитовой поверхности равна двум, из соотношений $\left(27_{1}\right)$ и $\left(27_{3}\right)$ вытекает, что

$$
B_{[b c]}^{a d}=-\frac{1}{4} \alpha^{h}{ }_{h} \delta_{b c}^{a d}, \quad B_{b c}{ }^{[a d]}=-\frac{1}{4} \alpha_{h}{ }^{h} \delta_{b c}^{a d},
$$

где $\delta_{b c}^{a d}=\delta_{b}^{a} \delta_{c}^{d}-\delta_{c}^{a} \delta_{b}^{d}$.

Автодуальные обобшенные эрмитовы поверхности. Пусть $M$ - автодуальная обобщенная эрмитова поверхность. В этом случае

$$
W(\theta)=0 \quad\left(\theta \in \Lambda_{-}(M)\right) \text {, т.е. } W_{i j c d} \theta^{c d}+2 W_{i j c \hat{d}} \theta^{c \hat{d}}+W_{i j \hat{c} \hat{d}} \theta^{\hat{c} \hat{d}}=0 .
$$

С учетом леммы 1 эти соотношения перепишутся в виде

$$
i y W_{i j 1 \hat{1}}-\alpha(x+i z) W_{i j 1 \hat{2}}+\alpha(x-i z) W_{i j 2 \hat{1}}+i \alpha y W_{i j 2 \hat{2}}=0 .
$$

Поскольку эти соотношения должны выполняться тождественно относительно формы $\theta$, они равносильны соотношениям

$$
W_{i j 1 \hat{1}}+\alpha W_{i j 2 \hat{2}}=0, \quad W_{i j 1 \hat{2}}=W_{i j 2 \hat{1}}=0,
$$

которые с учетом (1) и свойств симметрии тензора $W$ можно переписать в форме $W^{1}{ }_{1 i j}=W^{2}{ }_{2 i j}, W^{1}{ }_{2 i j}=W^{2}{ }_{1 i j}=0$, т.е.

$$
W_{b i j}^{a}=W_{i j} \delta_{b}^{a}, \quad \text { где } W_{i j}=\frac{1}{2} W^{h}{ }_{h i j} .
$$

В частности, с учетом $\left(23_{2}\right) W_{c d}=\frac{1}{2} W^{h}{ }_{h c d}=A_{h c d}^{h}$. Следовательно, $W_{c d}=$ $A_{h c d}^{h}=\frac{1}{2}\left(B_{c d}{ }^{r} B_{r h}{ }^{h}-B_{c d}{ }^{h} h\right)$. Прямой подсчет с учетом (24) и (27) показывает, что $W_{c d}=-\frac{1}{2} \alpha_{[c d]}$, и значит,

$$
W_{b c d}^{a}=-\frac{1}{2} \alpha_{[c d]} \delta_{b}^{a}
$$

С учетом $\left(23_{2}\right)$ очевидно, что это соотношение равносильно

$$
A_{b c d}^{a}=-\frac{1}{4} \alpha_{[c d]} \delta_{b}^{a}+\frac{1}{2} r_{b[c} \delta_{d]}^{a} .
$$

Заметим, что с учетом (1) непосредственно проверяется, что тождество $r_{c d}=0$ равносильно тому, что эндоморфизм Риччи коммутирует со структурным эндоморфизмом $J$ и, таким образом, мы получаем следуюшее 
ПРЕДЛОЖЕНИЕ 4. Эндоморфизм Риччи обобщенной эрмитовой поверхности Ј-линеен тогда и только тогда, когда

$$
r_{c d}=0 \text {. }
$$

Далее, из (28) вытекает, что $W^{a}{ }_{b c}{ }^{d}=W_{c}{ }^{d} \delta_{b}^{a}$, где с учетом (234)

$$
\begin{aligned}
2 W_{c}{ }^{d} & =W^{h}{ }_{h c}{ }^{d}=A_{h c}^{h d}-B^{h r}{ }_{c} B_{r h}{ }^{d}+\frac{1}{2}\left(r_{c}^{h} \delta_{h}^{d}+r_{h}^{d} \delta_{c}^{h}\right)-\frac{\varkappa}{6} \delta_{c}^{h} \delta_{h}^{d} \\
& =A_{h c}^{h d}+B^{h r}{ }_{c} B_{h r}{ }^{d}+r_{c}^{d}-\frac{\varkappa}{6} \delta_{c}^{d} .
\end{aligned}
$$

Используя (22), получим отсюда, что $2 W_{c}{ }^{d}=4 B^{h d}{ }_{[h c]}-\frac{\varkappa}{6} \delta_{c}^{d}$. С учетом замечания $4 B^{h d}{ }_{[h c]}=-\frac{1}{4} \alpha^{h}{ }_{h} \delta_{h}^{d}$, и следовательно, $W_{c}{ }^{d}=-\frac{1}{12}\left(6 \alpha^{h}{ }_{h}+\varkappa\right) \delta_{c}^{d}$, откуда

$$
W_{b c}^{a}{ }^{d}=-\frac{1}{12}\left(6 \alpha_{h}^{h}+\varkappa\right) \delta_{b}^{a} \delta_{c}^{d} .
$$

ЗАмЕчАниЕ 5 . Ввиду вешественности и свойств симметрии тензора $W$ имеем: $\bar{W}^{a}{ }_{b c}{ }^{d}=\varepsilon(a, b, c, d) W^{b}{ }_{a d}{ }^{c}$ и в силу $(32) \bar{\alpha}^{h}{ }_{h}=\alpha^{h}{ }_{h}$. Но поскольку $\bar{\alpha}_{h}{ }_{h}=\alpha_{h}{ }^{h}$,

$$
\alpha^{h}{ }_{h}=\alpha_{h}{ }^{h}=\alpha_{h}^{h} \text {. }
$$

Таким образом, $W_{b c}^{a}{ }^{d}=-\frac{1}{12}\left(6 \alpha_{h}^{h}+\varkappa\right) \delta_{b}^{a} \delta_{c}^{d}$. С учетом $\left(23_{4}\right)$ отсюда следует, что

$$
A_{b c}^{a d}=B^{a h}{ }_{c} B_{h b}{ }^{d}-\frac{1}{2}\left(r_{c}^{a} \delta_{b}^{d}+r_{b}^{d} \delta_{c}^{a}\right)+\frac{\varkappa}{6} \delta_{c}^{a} \delta_{b}^{d}-\frac{1}{12}\left(6 \alpha_{h}^{h}+\varkappa\right) \delta_{b}^{a} \delta_{c}^{d} .
$$

Заметим, что альтернируя это соотношение по индексам $b$ и $c$, с учетом (175) получим, что $B_{[b c]}^{a d}=-r_{[b}^{[a} \delta_{c]}^{d]}-\frac{1}{8}\left(2 \alpha_{h}^{h}-\varkappa\right) \delta_{b c}^{a d}$. С другой стороны, непосредственная проверка с учетом комплексной двумерности многообразия показывает, что

$$
r_{b}^{a} \delta_{c}^{d}+r_{c}^{d} \delta_{b}^{a}-r_{c}^{a} \delta_{b}^{d}-r_{b}^{d} \delta_{c}^{a}=\frac{\varkappa}{2} \delta_{b c}^{a d}
$$

и следовательно, $B_{[b c]}^{a d}=-\frac{1}{4} \alpha_{h}^{h} \delta_{b c}^{a d}$, что, впрочем, вытекает также из замечания 4. Далее, с учетом (175) и (33) имеем:

$$
\begin{aligned}
A_{b c}^{a d}=A_{[b c]}^{a d}+A_{(b c)}^{a d}=B_{[b c]}^{a d}+B^{a h}{ }_{[b} B_{c] h}{ }^{d}-B^{a h}{ }_{(c} B_{b) h}{ }^{d} & \\
& -r_{(c}^{(a} \delta_{b)}^{d)}+\frac{\varkappa}{12} \tilde{\delta}_{b c}^{a d}-\frac{1}{24}\left(6 \alpha_{h}^{h}-\varkappa\right) \tilde{\delta}_{b c}^{a d},
\end{aligned}
$$

где $\tilde{\delta}_{b c}^{a d}=\delta_{b}^{a} \delta_{c}^{d}+\delta_{c}^{a} \delta_{b}^{d}$, или, после очевидных упрощений,

$$
A_{b c}^{a d}=B_{[b c]}^{a d}+B^{a h}{ }_{c} B_{h b}{ }^{d}-r_{(b}^{(a} \delta_{c)}^{d)}-\frac{1}{24}\left(6 \alpha_{h}^{h}-\varkappa\right) \tilde{\delta}_{b c}^{a d} .
$$

Заметим, что эту формулу с учетом замечания 4 можно переписать в форме

$$
A_{b c}^{a d}=B^{a h}{ }_{c} B_{h b}{ }^{d}-r_{(b}^{(a} \delta_{c)}^{d)}-\frac{1}{2} \alpha_{h}^{h} \delta_{b}^{a} \delta_{c}^{d}+\frac{\varkappa}{24} \tilde{\delta}_{b c}^{a d} .
$$

Заметим также, что в силу (24)

$$
B^{a h}{ }_{c} B_{h b}{ }^{d}=\frac{1}{4}\left(\alpha^{a} \alpha_{c} \delta_{b}^{d}-\alpha^{a} \alpha_{b} \delta_{c}^{d}-\frac{1}{2}\|\omega\|^{2} \delta_{c}^{a} \delta_{b}^{d}+\alpha^{d} \alpha_{b} \delta_{c}^{a}\right) .
$$

Обратно, если имеет место соотношение (36), то непосредственный подсчет с учетом $\left(23_{4}\right)$ показывает, что $W^{a}{ }_{d c}{ }^{d}=-\frac{1}{12}\left(6 \alpha_{h}^{h}+\varkappa\right) \delta_{b}^{a} \delta_{c}^{d}$, и, таким образом, (32) эквивалентно (36). Тем самым доказана 
ТЕОРЕМА 2. Обобщенная эрмитова поверхность автодуальна тогда и только тогда, когда

1) $A_{b c d}^{a}=-\frac{1}{4} \alpha_{[c d]} \delta_{b}^{a}+\frac{1}{2} r_{b[c} \delta_{d]}^{a}$,

2) $A_{b c}^{a d}=B^{a h}{ }_{c} B_{h b}{ }^{d}-r_{(b}^{(a} \delta_{c)}^{d)}-\frac{1}{2} \alpha_{h}^{h} \delta_{b}^{a} \delta_{c}^{d}+\frac{\varkappa}{24} \tilde{\delta}_{b c}^{a d}$.

ЗАмечАниЕ 6. С учетом (22), (24) и (27) нетрудно проверить, что для обобщенных эрмитовых поверхностей

$$
r_{a b}=-\frac{1}{2}\left(\alpha_{a b}+\alpha_{b a}+\alpha_{a} \alpha_{b}\right)
$$

а следовательно, комплексная линейность тензора Риччи, т.е. соотношение (31), равносильна равенству $\alpha_{a b}+\alpha_{b a}+\alpha_{a} \alpha_{b}=0$.

\section{Обобшенные эрмитовы поверхности с $J$-инвариантным тензором кривизны.}

ОПРЕДЕЛЕНИЕ 3. $G A \mathscr{H}$-многообразие называется $R K$-многообразием, если его тензор Римана-Кристоффеля инвариантен относительно структурного эндоморфизма, т.е.

$$
\langle R(X, Y) Z, W\rangle=\langle R(J X, J Y) J Z, J W\rangle, \quad X, Y, Z, W \in \mathfrak{X}(M) .
$$

Это понятие обобшает соответствующее понятие классической эрмитовой геометрии [12].

ПРЕДЛОЖЕНИЕ 5. Обобщенная әрмитова поверхность является RK-многообразием тогда и только тогда, когда

$$
\alpha_{a b}=-\frac{1}{2} \alpha_{a} \alpha_{b}
$$

ДокАЗАТЕльство. Непосредственно проверяется, что условие (38), записанное на пространстве присоединенной $G$-структуры, примет вид $R_{\hat{a} b c d}=0$ или в силу $\left(18_{1}\right) \quad A_{b c d}^{a}=0$. Принимая во внимание $(21)$, перепишем его в виде $B_{c d}{ }^{a}{ }_{b}=B_{c d}{ }^{h} B_{h b}{ }^{a}$ или с учетом (24) и (27)

$$
\alpha_{b c} \delta_{d}^{a}-\alpha_{d c} \delta_{b}^{a}=-\frac{1}{2}\left(\alpha_{b} \alpha_{c} \delta_{d}^{a}-\alpha_{d} \alpha_{c} \delta_{b}^{a}\right)
$$

Свертывая по индексам $a$ и $d$, получим, что $\alpha_{b c}=-\frac{1}{2} \alpha_{b} \alpha_{c}$. Обратно, если верно это равенство, то, очевидно, справедливо (40), а значит, $A_{b c d}^{a}=0$, т.е. эрмитова поверхность является $R K$-многообразием. 
ЗАМЕчАниЕ 7 . Поскольку $\left\{\alpha_{a}, \alpha_{\hat{a}}\right\}$ - компоненты 1-формы (формы Ли), они удовлетворяют дифференциальньм уравнениям

$$
d \alpha_{i}+\alpha_{j} \omega_{i}^{j}=\alpha_{i, j} \omega^{j}
$$

где $\left\{\alpha_{i, j}\right\}$ - компоненты тензора $\nabla \omega$. В частности,

$$
d \alpha_{a}+\alpha_{b} \omega_{a}^{b}+\alpha_{\hat{b}} \omega^{\hat{b}}=\alpha_{a, b} \omega^{b}+\alpha_{a, \hat{b}} \omega^{\hat{b}}
$$

В силу (26) и замечания 3 эти соотношения можно переписать в форме

$$
\alpha_{a b} \omega^{b}+\alpha_{a \hat{b}} \omega^{\hat{b}}+\alpha^{b} B_{b a}{ }^{c} \omega_{c}=\alpha_{a, b} \omega^{b}+\alpha_{a, \hat{b}} \omega^{\hat{b}} .
$$

С учетом линейной независимости базисных форм получаем отсюда, что

$$
\text { 1) } \alpha_{a b}=\alpha_{a, b}, \quad \text { 2) } \alpha_{a}{ }^{b}=-\alpha^{c} B_{c a}{ }^{b}+\alpha_{a}{ }^{b} \text {. }
$$

С учетом этих соотношений имеем

$$
\begin{aligned}
\alpha_{h}^{h} & =-\alpha^{c} B_{c h}{ }^{h}+\alpha_{h}{ }^{h}=-\frac{1}{2} \alpha^{c} \alpha_{c} \delta_{h}^{h}+\frac{1}{2} \alpha^{c} \alpha_{h} \delta_{c}^{h}+\alpha_{h}{ }^{h} \\
& =-\frac{1}{4}\|\omega\|^{2}+\frac{1}{2} \alpha_{i, j} g^{i j}=-\frac{1}{4}\|\omega\|^{2}+\frac{1}{2} \delta \omega .
\end{aligned}
$$

Лемма 2. Внешний дифференциал формы Ли на обобщенной әрмитовой поверхности является антиавтодуальной 2-формой тогда и только тогда, когда на пространстве присоединенной $G$-структуры

$$
\text { 1) } \alpha_{a b}=\alpha_{b a}, \quad \text { 2) } \alpha_{h}^{h}=\alpha_{h}{ }^{h} \text {. }
$$

ДоКАЗАТЕЛЬСТво. В силу леммы 1 2-форма $d \omega$ на обобшенной эрмитовой поверхности антиавтодуальна тогда и только тогда, когда (с учетом ее вещественности)

$$
\text { 1) } \left.(d \omega)_{a b}=0, \quad 2\right)(d \omega)_{h}^{h}=0 .
$$

Поскольку $d \omega(X, Y)=\frac{1}{2}\left(\nabla_{X}(\omega) Y-\nabla_{Y}(\omega) X\right)$, эти соотношения можно переписать в форме

$$
\text { 1) } \alpha_{a, b}=\alpha_{b, a}, \quad \text { 2) } \alpha^{h}{ }_{, h}=\alpha_{h},^{h} .
$$

С учетом (41) эти соотношения, в свою очередь, равносильны (43).

ПрЕДЛОЖЕНИЕ 6. Автодуальная обобщенная эрмитова поверхность $М$ является $R K$-многообразием тогда и только тогда, когда ее тензор Риччи J-линеен, а внешний дифференциал ее формы Ли является антиавтодуальной формой. 
ДоказАТЕЛЬСтво. 1) Пусть $M-R K$-многообразие. Тогда в силу предложения $5 \alpha_{a b}=-\frac{1}{2} \alpha_{a} \alpha_{b}$. С учетом замечания 6 отсюда следует, что $r_{a b}=0$, т.е. тензор Риччи многообразия $M \quad J$-линеен. Кроме того, отсюда же следует первое из соотношений (43). Второе же из этих соотношений автоматически вьполняется в силу замечания 5 . С учетом леммы 2 заключаем, что форма $d \omega$ антиавтодуальна.

2) Обратно, если тензор Риччи многообразия $M J$-линеен, а форма $d \omega$ антиавтодуальна, то в силу $\left(43_{1}\right)$ и замечания 6 заключаем, что имеет место соотношение (39), и значит, $M$ является $R K$-многообразием.

Напомним [14], что (обобщенное) эрмитово многообразие называется локально конформно-келеровым, если его метрика локально допускает конформное преобразование в (обобщенную) келерову метрику (с тем же структурным оператором). Необходимьм и достаточньм условием локальной конформной келеровости многообразия является выполнение условий

$$
d \Omega=\omega \wedge \Omega, \quad d \omega=0 .
$$

ТЕоремА 3. Компактная автодуальная обобщенная әрмитова поверхность $M \quad c$ неизотропной 2-формой $d \omega$ (в частности, компактная автодуальная әрмитова поверхность) является $R K$-многообразием тогда и только тогда, когда $M$ - локально конформно-келерово многообразие $c$ J-линейным тензором Риччи.

ДокАЗАТЕльСтво. Пусть $M$ - компактная автодуальная обобщенная эрмитова поверхность с неизотропной 2-формой $d \omega$, являюшаяся $R K$-многообразием. По предложению 6 тензор Риччи этого многообразия $J$-линеен, а 2-форма $d \omega$ антиавтодуальна, и значит,

$$
(d \omega, d \omega)=\int_{M} d \omega \wedge(* d \omega)=-\int_{M} d \omega \wedge d \omega=-\int_{M} d(\omega \wedge d \omega)=0
$$

в силу классической теоремы Стокса. Ввиду неизотропности 2-формы $d \omega$ отсюда следует, что $d \omega=0$, т.е. форма Ли многообразия замкнута, и значит, $M-$ локально конформно-келерово многообразие. Обратное немедленно следует из предложения 6.

Обобшенные эрмитовы поверхности постоянной голоморфной секционной кривизны. Напомним [13], что голоморфной секционной кривизной почти эрмитова многообразия $M$ в направлении вектора $X \in \mathfrak{X}(M)$ называется кривизна в двумерном направлении $X \wedge J X$. Почти эрмитово многообразие $M$ назьвается многообразием (точечно) постоянной голоморфной секционной кривизны с, если

$$
\langle R(X, J X) X, J X\rangle=c\|X\|^{4}, \quad X \in \mathfrak{X}(M) .
$$

Запишем это соотношение на пространстве присоединенной $G$-структуры:

$$
\left(R_{\hat{a} b c \hat{d}}+c g_{\hat{a} b} g_{c \hat{d}}\right) X^{\hat{a}} X^{\hat{d}} X^{b} X^{d}=0 .
$$


Поскольку оно верно для любого $X \in \mathfrak{X}(M)$, оно равносильно соотношению $R^{(a)}{ }_{(b c)}{ }^{d)}=-\frac{c}{2} \tilde{\delta}_{c d}^{a b}$. Если $M-G \mathscr{H}$-многообразие, то с учетом $\left(18_{2}\right)$

$$
A_{(b c)}^{(a d)}=-\frac{c}{2} \tilde{\delta}_{b c}^{a d}+B_{(c}^{h(a} B_{b) h}{ }^{d)} .
$$

Заметим, что $A_{b c}^{a d}=A_{(b c)}^{(a d)}+A_{(b c)}^{[a d]}+A_{[b c]}^{(a d)}+A_{[b c]}^{[a d d]}=A_{(b c)}^{(a d)}+A_{(b c)}^{[a d]}+A_{[b c]}^{a d}$. С учетом $\left(17_{5}\right),\left(17_{6}\right)$ и $(44)$ получаем:

$$
\begin{aligned}
A_{b c}^{a d} & =\frac{c}{2} \tilde{\delta}_{b c}^{a d}+B^{h(a}{ }_{(c} B_{b)}{ }^{d)}-B_{h(b}{ }^{[a} B^{d] h}{ }_{c)}+B_{[b c]}^{a d}-B_{[b}^{h a} B_{c] h}{ }^{d} \\
& =\frac{c}{2} \tilde{\delta}_{b c}^{a d}+B^{h a}{ }_{h(b}\left({ }^{(a} B^{d) h}{ }_{c)}-B_{h(b}{ }^{a}{ }^{a} B^{d] h}{ }_{c)}+B_{[b c]}^{a d}-B_{[b}^{h a} B_{c] h}{ }^{d}\right. \\
& =\frac{c}{2} \tilde{\delta}_{b c}^{a d}+B_{h(b}{ }^{d} B^{a h}{ }_{c)}-B_{h[c}{ }^{d} B^{a h}{ }_{b]}+B^{a d}{ }_{[b c]} \\
& =\frac{c}{2} \tilde{\delta}_{b c}^{a d}+B_{h b}{ }^{d} B^{a h}{ }_{c}+B^{a d}{ }_{[b c]} .
\end{aligned}
$$

Тем самым доказана

ТЕОРема 4. Обобщенное эрмитово многообразие является многообразием точечно постоянной голоморфной секиионной кривизны тогда и только тогда, когда на пространстве присоединенной $G$-структуры выполняется соотношение

$$
A_{b c}^{a d}=B_{[b c]}^{a d}+B_{c}^{a h} B_{h b}{ }^{d}-\frac{c}{2} \tilde{\delta}_{b c}^{a d} .
$$

Пусть теперь $M$ - автодуальная эрмитова поверхность точечно постоянной голоморфной секционной кривизны $c$ с $J$-линейным тензором Риччи. С учетом формулы (35) и теоремы 4 для нее вьполняется соотношение

$$
r_{(b}^{(a} \delta_{c)}^{d)}=\frac{1}{24}\left(\varkappa-6 \alpha_{h}^{h}+12 c\right) \tilde{\delta}_{b c}^{a d} .
$$

Свернем его по индексам $d$ и $c$ :

$$
r_{b}^{a}=\frac{1}{8}\left(-6 \alpha_{h}^{h}+12 c\right) \delta_{b}^{a} .
$$

В свою очередь, сворачивая это соотношение по индексам $a$ и $b$, получим, что

$$
\varkappa=6 c-3 \alpha_{h}^{h} \text {. }
$$

Поскольку в силу $J$-линейности тензора Риччи $r_{a b}=0$, имеем отсюда $r_{j}^{i}=\frac{\varkappa}{4} \delta_{j}^{i}$, т.е. $M$ - многообразие Эйнштейна.

Обратно, если автодуальная обобщенная эрмитова поверхность $M$ является многообразием Эйнштейна, то с учетом (35) и соотношения $r_{b}^{a}=\frac{\varkappa}{4} \delta_{b}^{a}$ имеем:

$$
\begin{aligned}
A_{b c}^{a d} & =B_{[b c]}^{a d}+B^{a h}{ }_{c} B_{h b}{ }^{d}-\frac{\varkappa}{4} \alpha_{(b}^{(a} \delta_{c)}^{d)}-\frac{1}{24}\left(6 \alpha_{h}^{h}-\varkappa\right) \tilde{\delta}_{b c}^{a d} \\
& =B_{[b c]}^{a d}+B^{a h}{ }_{c} B_{h b}{ }^{d}-\frac{1}{12}\left(3 \alpha_{h}^{h}+\varkappa\right) \tilde{\delta}_{b c}^{a d}
\end{aligned}
$$

и значит, $M$ - многообразие точечно постоянной голоморфной секционной кривизны $c=\frac{1}{6}\left(3 \alpha_{h}^{h}+\varkappa\right)$. С учетом (42) мы получаем следуюший результат. 
ТЕОРема 5. Автодуальная обобщенная эрмитова поверхность является многообразием Эйнштейна тогда и только тогда, когда она является многообразием точечно постоянной голоморфной секичонной кривизнь с с J-линейным тензором Риччи. При этом

$$
c=\frac{\varkappa}{6}-\frac{1}{8}\|\omega\|^{2}+\frac{1}{4} \delta \omega .
$$

Этот результат существенно обобщает и усиливает известный результат Коды [6], утверждающий, что автодуальная эрмитова поверхность Эйнштейна является многообразием точечно постоянной голоморфной секционной кривизны.

ТЕОРема 6. Компактная автодуальная әрмитова поверхность $M$ точечно постоянной неотрицательной голоморфной секционной кривизнь и неположительной скалярной кривизны локально голоморфно изометрична пространству $\mathbb{C}^{2}$, снабэсенному канонической келеровой структурой.

ДокАЗАтЕЛЬство. Пусть $\xi$-вектор Ли, т.е. вектор, дуальный форме Ли, многообразия $M$. По определению $\delta \omega=\operatorname{div} \xi$ и в силу (46) $\operatorname{div} \xi=\frac{1}{2}\|\omega\|^{2}-\frac{2}{3} \varkappa+4 c$. Поэтому если $\varkappa \leqslant 0, c \geqslant 0$, то $\operatorname{div} \xi \geqslant 0$. Следовательно, если $M$ компактно, то

$$
\int_{M} \operatorname{div} \xi \geqslant 0
$$

и в силу классической теоремы Грина $\operatorname{div} \xi=0$, т.е. $\frac{1}{2}\|\omega\|^{2}-\frac{2}{3} \varkappa+4 \mathrm{c}=0$, и значит, $\omega=\varkappa=c=0$. В частности, $M-4$-мерное келерово многообразие нулевой голоморфной секционной кривизны. Хорошо известно [13], что такое многообразие локально голоморфно изометрично пространству $\mathbb{C}^{2}$, снабженному канонической келеровой структурой.

Антиавтодуальные обобщенные эрмитовы поверхности. Пусть $M$ антиавтодуальная обобщенная эрмитова поверхность. В этом случае

$$
W(\theta)=0 \quad\left(\theta \in \Lambda_{+}(M)\right), \quad \text { т.e. } W_{i j c d} \theta^{c d}+2 W_{i j c \hat{d}} \theta^{c \hat{d}}+W_{i j \hat{c} \hat{d}} \theta^{\hat{c} \hat{d}}=0 .
$$

С учетом леммы 1 эти соотношения перепишутся в виде

$$
-\alpha(x+i z) W_{i j 12}+i y W_{i j 1 \hat{1}}-i \alpha y W_{i j 2 \hat{2}}-\alpha(x-i z) W_{i j \hat{1} \hat{2}}=0 .
$$

Поскольку эти соотношения должны выполняться тождественно относительно формы $\theta$, они равносильны соотношениям

$$
W_{i j 12}=W_{i j \hat{1} \hat{2}}=0, \quad W_{i j 1 \hat{1}}-\alpha W_{i j 2 \hat{2}}=0
$$

В силу свойств симметрии тензора $W$ эти соотношения можно переписать в форме

$$
\text { 1) } W_{a b c d}=0, \text { 2) } W_{\hat{a} b c d}=0, \text { 3) } W_{\hat{a} \hat{b} c d}=0, \text { 4) } W_{h c \hat{d}}^{h}=0 \text {. }
$$


Соотношение $\left(47_{1}\right)$ совпадает с $\left(23_{1}\right)$. Соотношение $\left(47_{2}\right)$ в силу $\left(23_{2}\right)$ равносильно соотношению

$$
A_{b c d}^{a}=-\frac{1}{4}\left(r_{b d} \delta_{c}^{a}-r_{b c} \delta_{d}^{a}\right)
$$

или с учетом (21) соотношению

$$
B_{c d}{ }^{h} B_{h b}^{a}-B_{c d}{ }^{a} b=-\frac{1}{2}\left(r_{b d} \delta_{c}^{a}-r_{b c} \delta_{d}^{a}\right) .
$$

Используя (24) и (27), после очевидных упрощений с учетом замечания 6 получим:

$$
\left(\alpha_{b c}-\alpha_{c b}\right) \delta_{d}^{a}=\left(\alpha_{b d}-\alpha_{d b}\right) \delta_{c}^{a} .
$$

Свертывая это соотношение по индексам $a$ и $d$, получим, что

$$
\alpha_{b c}=\alpha_{c b} .
$$

Обратно, если выполняется (49), то вьполняется и (48), а значит, и $\left(47_{2}\right)$.

Соотношение $\left(47_{3}\right)$ в силу $\left(23_{3}\right)$ равносильно соотношению

$$
B_{[c d]}^{a b}=\frac{1}{4}\left(r_{c}^{a} \delta_{d}^{b}+r_{d}^{b} \delta_{c}^{a}-r_{d}^{a} \delta_{c}^{b}-r_{c}^{b} \delta_{d}^{a}\right)+\frac{\varkappa}{12} \delta_{d c}^{a b}
$$

или с учетом замечания 4 и соотношения (34) равносильно соотношению

$$
\alpha_{h}^{h}=-\frac{\varkappa}{6} .
$$

В частности,

$$
\alpha_{h}{ }^{h}=\bar{\alpha}^{h}{ }_{h}=\alpha_{h}^{h}=\alpha_{h}^{h} .
$$

Соотношение $\left(47_{4}\right)$ в силу $\left(23_{4}\right)$ равносильно соотношению

$$
A_{h c}^{h d}+B^{r h}{ }_{c} B_{r h}{ }^{d}+r_{c}^{d}-\frac{\varkappa}{6} \delta_{c}^{d}=0 .
$$

С учетом (22) эти соотношения можно переписать в форме

$$
4 B_{[h c]}^{h d}-\frac{\varkappa}{6} \delta_{c}^{d}=0,
$$

и с учетом замечания 4

$$
\alpha_{h}^{h}=-\frac{\varkappa}{6} .
$$

Объединяя полученные данные, мы видим, что необходимым и достаточным условием антиавтодуальности обобшенной эрмитовой поверхности является выполнение соотношений

$$
\text { 1) } \alpha_{a b}=\alpha_{b a}, \quad \text { 2) } \varkappa=-6 \alpha_{h}^{h} .
$$

Заметим, что в силу (42) второе из этих соотношений равносильно соотношению

$$
\delta \omega=\frac{1}{2}\|\omega\|^{2}-\frac{\varkappa}{3} .
$$

Из соотношений (52) с учетом леммы 2 , соотношений (50) и (53) получаем следующий результат. 
ТЕОРема 7. Обобщенная әрмитова поверхность М антиавтодуальна тогда и только тогда, когда

1) внешний дифференциал ее формы Ли $\omega$ является антиавтодуальной 2-формой,

2) $\delta \omega=\frac{1}{2}\|\omega\|^{2}-\frac{\varkappa}{3}$.

Этот результат существенно обобщает известный критерий Ито [9] антиавтодуальности келеровых поверхностей, а также соответствующий результат одного из авторов для обобщенных келеровых поверхностей [10]. С помошью теоремы 7 нетрудно получить следуюшие результаты.

ТЕорема 8. Компактная обобщенная әрмитова поверхность $M$ с неизотропной 2-формой $d \omega$ (в частности, компактная эрмитова поверхность) антиавтодуальна тогда и только тогда, когда

1) $M$ - локально конформно-келерово многообразие,

2) $\delta \omega=\frac{1}{2}\|\omega\|^{2}-\frac{\varkappa}{3}$.

ДокАЗАТЕЛЬСтво. Пусть $M$ - компактная антиавтодуальная эрмитова поверхность. По теореме 7,2 -форма $d \omega$ антиавтодуальна, и значит,

$$
(d \omega, d \omega)=\int_{M} d \omega \wedge(* d \omega)=-\int_{M} d \omega \wedge d \omega=-\int_{M} d(\omega \wedge d \omega)=0
$$

в силу классической теоремы Стокса. Ввиду неизотропности 2-формы $d \omega$ отсюда следует, что $d \omega=0$, т.е. форма Ли многообразия замкнута, и значит, $M$ - локально конформно-келерово многообразие. Обратное немедленно следует из теоремы 7.

Этот результат существенно обобщает и проясняет известный критерий Коды антиавтодуальности компактных эрмитовых поверхностей [6].

ТЕОРема 9. Компактная антиавтодуальная эрмитова поверхность неположительной скалярной кривизны является келеровым многообразием нулевой скалярной кривизны.

ДоКАЗАТЕЛЬСТво вполне аналогично доказательству теоремы 6 с учетом соотношения (53).

ТЕОРемА 10. Метрика компактной антиавтодуальной эрмитовой поверхности локально конформна метрике нулевой скалярной кривизны.

ДокАЗАТЕЛЬСтво. Пусть $M$ - компактная антиавтодуальная эрмитова поверхность. По теореме 8 она является локально конформно-келеровым многообразием. Рассмотрим локальную келерову метрику на $M$, конформную исходной метрике. Поскольку свойство антиавтодуальности инвариантно относительно конформных преобразований метрики, эта метрика антиавтодуальна, а следовательно, в силу (53) ее скалярная кривизна равна нулю. 
Паракелеровы обобщенные эрмитовы поверхности. Напомним, что почти эрмитово многообразие $(M, J, g)$ называется паракелеровым, если для него выполняется тождество Келера

$$
R(J X, J Y)=R(X, Y), \quad X, Y \in \mathfrak{X}(M) .
$$

С учетом классических свойств симметрии тензора $R$ легко заметить, что это условие равносильно комплексной линейности операторов кривизны, т.е.

$$
J \circ R(X, Y)=R(X, Y) \circ J, \quad X, Y \in \mathfrak{X}(M) .
$$

Обобщая это понятие, введем

ОПреДЕЛЕНИЕ 4. GAभ̆-многообразие называется паракелеровым, если его операторы кривизны $J$-линейны, т.е. выполняются соотношения (54).

ПрЕДЛОЖЕНИЕ 7. Обобщенная әрмитова поверхность $M$ является паракелеровым многообразием тогда и только тогда, когда

1) $M$ является $R K$-многообразием,

2) $\delta \omega=\frac{1}{2}\|\omega\|^{2}$.

ДокАЗАТЕЛЬСтво. Непосредственная проверка с учетом формулы (1) показывает, что условие (54) равносильно соотношениям $R_{i j a b}=0$ на пространстве присоединенной $G$-структуры. Эти соотношения можно сгруппировать так:

$$
\text { 1) } R_{a b c d}=0, \quad \text { 2) } R_{\hat{a} b c d}=0, \quad \text { 3) } R_{\hat{a} \hat{b} c d}=0
$$

Первая группа этих соотношений выполняется автоматически в силу $\left(20_{1}\right)$, вторая, как мы видели при доказательстве предложения 5 , равносильна тому, что $M$ является $R K$-многообразием, а третья в силу $\left(20_{2}\right)$ и замечания 4 равносильна тому, что $\alpha_{h}{ }^{h}=0$, и в силу (42) она равносильна тому, что $\delta \omega=\frac{1}{2}\|\omega\|^{2}$.

СлЕДСТвИЕ 1. Компактная паракелерова әрмитова поверхность является келеровым многообразием.

ДокАЗАТЕЛЬСТво вполне аналогично доказательству теоремы 6 .

С учетом теоремы 7 получаем также

СлЕДСТВИЕ 2. Антиавтодуальная обобщенная әрмитова поверхность является паракелеровым многообразием тогда и только тогда, когда она является RK-многообразием нулевой скалярной кривизны.

Заметим, что в силу тождества $\left(52_{1}\right)$, замечания 6 и предложения 5 справедливо

ПРЕДЛОЖЕНИЕ 8. Антиавтодуальная обобщенная эрмитова поверхность является RK-многообразием тогда и только тогда, когда ее тензор Риччи J-линеен. 
Форма Риччи обобщенных эрмитовых поверхностей. Пусть $(M, J, g)-$ $G A \mathscr{H}$-многообразие. Рассмотрим тензор

$$
\rho(X, Y)=\frac{1}{2}\left\langle\left(J^{3} \circ r+r \circ J^{3}\right) X, Y\right\rangle, \quad X, Y \in \mathfrak{X}(M) .
$$

В случае, когда $M$ - классическое почти эрмитово многообразие с $J$-линейньм тензором Риччи, например, келерово многообразие, этот тензор вычисляется по формуле $\rho(X, Y)=\langle r X, J Y\rangle$. В частности, в случае келерова многообразия он совпадает с классической формой Риччи [13]. Назовем его формой Риччи $G A \mathscr{H}$-многообразия. Заметим, что на пространстве присоединенной $G$-структуры компоненты формы Риччи имеют вид:

$$
\begin{aligned}
\rho_{a b} & =\rho\left(\varepsilon_{a}, \varepsilon_{b}\right)=\frac{1}{2}\left\langle\left(J^{3}\right)_{a}^{c} r_{c}^{h} \varepsilon_{h}+\left(J^{3}\right)_{a}^{c} r_{c}^{\hat{h}} \varepsilon_{\hat{h}}+r_{a}^{c}\left(J^{3}\right)_{c}^{h} \varepsilon_{h}+r_{a}^{\hat{c}}\left(J^{3}\right)_{\hat{c}}^{\hat{h}} \varepsilon_{\hat{h}}, \varepsilon_{b}\right\rangle \\
& =\frac{1}{2}\left\langle i^{3} r_{a}^{h} \varepsilon_{h}+i^{3} r_{a}^{\hat{h}} \varepsilon_{\hat{h}}+i^{3} r_{a}^{h} \varepsilon_{h}-i^{3} r_{a}^{\hat{h}} \varepsilon_{\hat{h}}, \varepsilon_{b}\right\rangle=i^{3} r_{a}^{h}\left\langle\varepsilon_{h}, \varepsilon_{b}\right\rangle=0 .
\end{aligned}
$$

Аналогично, $\rho_{a \hat{b}}=\rho\left(\varepsilon_{a}, \varepsilon_{\hat{b}}\right)=i^{3} r_{a}^{h}\left\langle\varepsilon_{h}, \varepsilon_{\hat{b}}\right\rangle=i^{3}\left\langle r_{a}^{h} \varepsilon_{h}, \varepsilon_{\hat{b}}\right\rangle=i^{3} r_{a \hat{b}}$. Кроме того, $\rho_{\hat{a} \hat{b}}=\bar{\rho}_{a b}=0, \rho_{\hat{a} b}=-\rho_{b \hat{a}}=-i^{3} r_{b \hat{a}}$. Следовательно, в локальной записи

$$
\rho=2 i^{3} r_{a}^{b} \omega^{a} \wedge \omega_{b}
$$

ТЕОРЕМА 11. Пусть $M$ - автодуальная обобщенная әрмитова поверхность. Тогда следующие утверждения әквивалентнь:

1) Форма Риччи многообразия $M$ автодуальна,

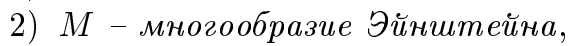

3) $M$ - многообразие точечно постоянной голоморфной секционной кривизныл.

ДокАЗАТЕЛЬСтво. Эквивалентность первого и второго утверждений непосредственно следует из леммы 1 , предложения 4 (или формулы (29)) и соотношения (55). Эквивалентность второго и третьего утверждений составляет содержание теоремы 5 .

ТЕОРЕМА 12. Обобщенная әрмитова поверхность является многообразием нулевой скалярной кривизнь тогда и только тогда, когда ее форма Риччи антиавтодуальна.

ДоКАЗАТЕЛЬСТво немедленно следует из леммы 1 и соотношения (55).

Докажем в заключение следующую теорему.

ТЕОРема 13. Антиавтодуальная обобщенная әрмитова поверхность точечно постоянной голоморфной секиионной кривизны имеет автодуальную форму Риччи. 
ДокАЗАТЕЛЬСТво. Пусть $M$ - антиавтодуальная обобщенная эрмитова поверхность точечно постоянной голоморфной секционной кривизны $c$. В силу теоремы 5 , замечания 4 и соотношения $\left(52_{2}\right)$ для нее

$$
A_{b c}^{a d}=\frac{\varkappa}{24} \delta_{b c}^{a d}+B^{a h}{ }_{c} B_{h b}{ }^{d}-\frac{c}{2} \tilde{\delta}_{b c}^{a d},
$$

и значит,

$$
A_{h c}^{h d}=\left(\frac{\varkappa}{24}-\frac{3 c}{2}\right) \delta_{c}^{d}-B^{r h}{ }_{c} B_{r h}{ }^{d} .
$$

С другой стороны, в силу (51)

$$
A_{h c}^{h d}=-B^{r h}{ }_{c} B_{r h}{ }^{d}-r_{c}^{d}+\frac{\varkappa}{6} \delta_{c}^{d}
$$

Сравнивая эти соотношения, получаем, что

$$
r_{c}^{d}=\left(\frac{\varkappa}{8}+\frac{3 c}{2}\right) \delta_{c}^{d}
$$

Свернем это соотношение по индексам $c$ и $d: \frac{\varkappa}{2}=\frac{\varkappa}{4}+3 c$, откуда $\varkappa=12 c$, и значит, $r_{c}^{d}=\frac{\varkappa}{4} \delta_{c}^{d}$. С учетом леммы 1 и (55) получаем, что форма Риччи многообразия $M$ автодуальна.

С учетом предложения 8 получаем

СлЕДСТВИЕ 1. Антиавтодуальная обобщенная әрмитова поверхность точечно постоянной голоморфной секционной кривизны является многообразием Эйнштейна тогда и только тогда, когда она является $R K$-многообразиe⿻..

СлЕДСТвИЕ 2. Пусть $M$ - антиавтодуальная обобщенная әрмитова поверхность точечно постоянной голоморфной секционной кривизны. Тогда следующие условия эквивалентни:

1) $M$ - паракелерово многообразие,

2) $M$ - риччи-плоское многообразие,

3) $M-$ многообразие нулевой скалярной кривизньц.

ДоКАЗАТЕЛЬСТво. Согласно следствию 2 предложения $7, M$ - паракелерово многообразие тогда и только тогда, когда $M-R K$-многообразие нулевой скалярной кривизны. С другой стороны, по только что доказанному $M$ является многообразием Эйнштейна и, значит, условие $\varkappa=0$ равносильно тому, что многообразие $M$ риччи-плоско. Наконец, если $M$ риччи-плоско, то, в частности, его тензор Риччи $J$-линеен, и по предложению $8, M$ является $R K$-многообразием. Согласно следствию 2 предложения $7 M$ - паракелерово многообразие. 
Обобщенные многообразия Хопфа. Напомним, что эрмитово многообразие с параллельной формой Ли называется обобщенным многообразием Хопфа. Мы перенесем эту терминологию и на случай обобщенных эрмитовых многообразий. Обобшенные многообразия Хопфа играют важную роль при построении нелинейных сигма-моделей в квантовой теории поля, и их изучению посвящено значительное число работ (см., например, [14]).

Пусть $M-G A \mathscr{H}$-многообразие. С учетом (42) мы видим, что условие $\nabla \omega=0$ равносильно соотношениям

$$
\text { 1) } \alpha_{a b}=0, \quad \text { 2) } \alpha_{a}{ }^{b}=-\alpha^{c} B_{c a}{ }^{b} \text {. }
$$

В частности, для обобщенных эрмитовых поверхностей с учетом (24) эти соотношения примут вид

$$
\text { 1) } \alpha_{a b}=0, \quad \text { 2) } \alpha_{a}{ }^{b}=-\frac{1}{4}\|\omega\|^{2} \delta_{a}^{b}+\frac{1}{2} \alpha^{b} \alpha_{a} .
$$

Заметим, что поскольку форма Ли обобщенного многообразия Хопфа замкнута, условия антиавтодуальности такой поверхности в силу теоремы 7 примут вид

$$
\delta \omega=\frac{1}{2}\|\omega\|^{2}-\frac{\varkappa}{3}
$$

С другой стороны, параллельность формы Ли влечет ее козамкнутость, в силу чего справедлива

ТЕОРема 14. Обобщенная әрмитова поверхность, являющаяся обобщенныц многообразием Хопфа, антиавтодуальна тогда и только тогда, когда

$$
\varkappa=\frac{3}{2}\|\omega\|^{2}
$$

СлЕДСТВИЕ 1. Связная антиавтодуальная обобщенная әрмитова поверхность, являющаяся обобщенныц многообразием Хопфа, является многообразием постоянной скалярной кривизны.

ДокАЗАТЕЛЬСТво. Пусть $X$ - векторное поле на таком многообразии. Тогда

$$
d \varkappa(X)=X(\varkappa)=\frac{3}{2} X\left(\|\omega\|^{2}\right)=\frac{3}{2} X(\langle\omega, \omega\rangle)=3\left\langle\nabla_{X} \omega, \omega\right\rangle=0 .
$$

Следовательно, $d \varkappa=0$, т.е. $\varkappa=$ const. 
СЛЕДСТВИЕ 2. Связная антиавтодуальная эрмитова поверхность $M$, являющаяся обобщенным многообразием Хопфа, является многообразием постоянной неотрицательной скалярной кривизны. При этом ее скалярная кривизна равна нулю тогда и только тогда, когда $M$ - келерово многообразиe.

Пусть теперь $M$ - автодуальная обобщенная эрмитова поверхность, являющаяся обобшенньм многообразием Хопфа. В силу теоремы 2 и соотношения $\left(41_{1}\right)$ в этом случае $A_{b c d}^{a}=\frac{1}{2} r_{b[c} \delta_{d]}^{a}$ и в силу $(21)$ и $\left(56_{1}\right) B_{c d}{ }^{h} B_{h b}{ }^{a}=r_{b[c} \delta_{d]}^{a}$. После упрощений с учетом (24) и (27) находим, что $\alpha_{b} \alpha_{[d} \delta_{c]}^{a}=2 r_{b[c} \delta_{d]}^{a}$. Свертывая это соотношение по индексам $a$ и $d$, получим, что $r_{b c}=-\frac{1}{2} \alpha_{b} \alpha_{c}$. В частности, если $M$ - $R K$-многообразие, то в силу предложения $8 r_{b c}=0$, и значит, $\alpha_{b} \alpha_{c}=0$. Следовательно, $\alpha_{c}=0, a^{c}=\bar{\alpha}_{c}=0$, т.е. $\omega=0$, и значит, $M-$ келерово многообразие. Поскольку оно автодуально, в силу теоремы 2 в [10] имеем:

$$
A_{b c}^{a d}=t_{(b}^{(a} \delta_{c)}^{d)}, \quad \text { где } \quad t_{b}^{a}=-r_{b}^{a}+\frac{\varkappa}{12} \delta_{b}^{a}
$$

Обратно, если $M-4$-мерное келерово многообразие, для которого выполнено это соотношение, то в силу той же теоремы $M$ автодуально. Доказана

ТЕОРема 15. Обобщенная эрмитова $R K$-поверхность $M$, являющаяся обобщенным многообразием Хопфа, автодуальна тогда и только тогда, когда

$$
A_{b c}^{a d}=t_{(b}^{(a} \delta_{c)}^{d)}
$$

где с необходимостью $t_{b}^{a}=-r_{b}^{a}+\frac{\varkappa}{12} \delta_{b}^{a}$.

Следуя [10], назовем обобщенную эрмитову поверхность неисключительной, если ее эндоморфизм Риччи имеет по крайней мере один неизотропный собственный вектор в каждой ее точке. Примером такой поверхности, очевидно, является произвольная эрмитова поверхность. С учетом [10, теорема 3] получаем

СлЕДСТВИЕ 1. Всякая автодуальная неисключительная обобщенная эрмитова RK-поверхность, являющаяся обобщенным многообразием Хопфа постоянной скалярной кривизны, локально голоморфно изометрична одному из следующих многообразий:

1) комплексной плоскости $\mathbb{C}^{2}$,

2) комплексной проективной плоскости $\mathbb{C} P^{2}$,

3) комплексной гиперболической плоскости $\mathbb{C H}^{2}$,

4) двойному евклидову пространству $\mathbb{R}^{2} \otimes \mathbb{R}^{2}$,

5) пространству нуль-пар $\mathbb{R} P^{2} \square \mathbb{R} P^{2}$ вещественной проективной плоскости,

6) многообразию $S_{\lambda}^{2} \times S_{-\lambda}^{2}$,

снабэсенных канонической келеровой структурой классического либо, соответственно, гиперболического типа. Здесь $S_{\mu}^{2}$ обозначает двумерное многообразие постоянной кривизны $\mu$. 
СлЕДСТВИЕ 2. Автодуальная әрмитова RK-поверхность, являющаяся обобщенным многообразие Хопфа постоянной скалярной кривизны, локально голоморфно изометрична одному из следующих многообразий:
1) $\mathbb{C}^{2}$
2) $\mathbb{C} P^{2}$,
3) $\mathbb{C} H^{2}$,
4) $S_{\lambda}^{2} \times S_{-\lambda}^{2}$,

снабэенньх канонической келеровой структурой.

СлЕДСТВИЕ 3. Компактная автодуальная эрмитова $R K$-поверхность, являющаяся обобщенным многообразием Хопфа, голоморфно изометрично накрывается одним из следующих многообразий:
1) $\mathbb{C}^{2}$
2) $\mathbb{C} P^{2}$,
3) $\mathbb{C} H^{2}$,
4) $S_{\lambda}^{2} \times S_{-\lambda}^{2}$,

снабэкенных канонической келеровой структурой.

Этот результат дает полное решение проблемы Чена в классе компактных автодуальных эрмитовых $R K$-поверхностей, являющихся обобщенными многообразиями Хопфа.

\section{Список литературы}

1. Tricerri F., Vaisman I. On some 2-dimensional Hermitian manifolds // Math. Z. 1986. V. 192. P. 205-216.

2. Vaisman I. Some curvature properties of complex surfaces // Ann. Math. Pura Appl. (4). 1982. V. 32. P. 1-18.

3. De Andres L.C., Cordero L.A., Fernandes M., Mencia J.J. Examples of four-dimensional compact locally conformal Kaehler solvmanifolds // Geom. Dedicata. 1989. V. 29. № 2. P. 227-232.

4. Atiyah M.F., Hitchin N. J., Singer I. M. Self-duality in four-dimensional Riemannian geometry // Proc. Roy. Soc. London. Ser. A. 1978. V. 362. P. 425-461.

5. Chen B. Y. Some topological obstructions to Bochner-Kaehler metrics and their applications // J. Differential Geom. 1978. V. 13. P. 547-558.

6. Koda T. Self-dual and anti-self-dual Hermitian surfaces // Kodai Math. J. 1987. V. 10. P. 335-342.

7. Bourguignon J.-P. Les variétés de dimension 4 à signature non nulle dont la courbure est harmonique sont d'Einstein // Invent. Math. 1981. V. 63. P. 263-286.

8. Derdzinski A. Self-dual Kähler manifolds and Einstein manifolds of dimensional four // Compos. Math. 1983. V. 49. P. 405-433.

9. Itoh M. Self-duality of Kähler surfaces // Compos. Math. 1984. V. 51. P. 265-273.

10. Арсеньева O. E. Автодуальная геометрия обобщенных келеровых многообразий // Матем. сб. 1993. Т. 184. № 8. С. 137-148.

11. Кириченко В. $\Phi$. Методы обобщенной эрмитовой геометрии в теории почти контактных многообразий // Итоги науки и техники. Проблемы геометрии. Т. 18. М.: ВИНИТИ, 1986. C. $25-72$.

12. Vanhecke L. Almost Hermitian manifolds with $J$-invariant Riemann curvature tensor // Rend. Sem. Mat. Univ. Politec. Torino. 1975-1976. V. 34. P. 487-498.

13. Кобалии ШШ., Номидзу К. Основы дифференциальной геометрии. Т. 1, 2: Мир, 1980.

14. Vaisman I. Locally conformal Kähler manifolds with parallel Lee form // Rend. Mat. (6). 1979. V. 12. № 2. P. 263-284. 